\title{
ON REPLACING PROPER DEHN MAPS WITH PROPER EMBEDDINGS
}

\author{
BY \\ C. D. FEUSTEL
}

\begin{abstract}
In this paper we develop algebraic and geometric conditions which imply that a given proper Dehn map can be replaced by an embedding. The embedding, whose existence is implied by our theorem, retains most of the algebraic and geometric properties required in the original proper Dehn map.
\end{abstract}

I. Preliminaries. In this paper all spaces will be simplicial complexes and all maps will be piecewise linear. The boundary, closure, and interior of a subset $X$ of a space $Y$ will be denoted by bd $(X), \operatorname{cl}(X)$ and int $(X)$, respectively. A proper map $f$, taking a space $X$ into a space $Y$, is a map such that

$$
f \text { bd }(X)=\operatorname{bd}(Y) \cap f(X) .
$$

A Dehn map $f$, taking a compact, bounded, two-manifold $F$ into a 3-manifold $M$, is a map such that

(1) bd $(F)=f^{-1} f$ bd $(F)$;

(2) $f \mid$ bd $(F)$ is a homeomorphism.

All unlabeled homomorphisms are natural maps induced by inclusion.

We shall consider the problem of replacing a proper Dehn map of a compact, connected, orientable, bounded surface $F$ into a compact, (necessarily) bounded, irreducible, orientable 3-manifold $M$ with an embedding. As will be pointed out later, the requirement of orientability may not be strictly necessary.

The author would like to thank J. Gross for a number of helpful conversations.

In [3] Papakyriakopoulos proved Dehn's lemma, that is, a proper Dehn map $f$ of a disk $\mathscr{D}$ into a 3-manifold $M$ can be replaced by a proper embedding $g$ of $\mathscr{D}$ into $M$ such that $g$ bd $(\mathscr{D})=f$ bd $(\mathscr{D})$. A natural question to consider is the following: Let $f: F \rightarrow M$ be a proper Dehn map of a compact, connected, bounded, orientable surface $F$ into a compact 3-manifold $M$. Does there always exist a proper embedding $g: F_{1} \rightarrow M$ such that

(1) $g\left(\right.$ bd $\left.\left(F_{1}\right)\right) \subseteq f($ bd $(F))$;

(2) genus $\left(F_{1}\right) \leqq$ genus $(F)$ ? (Question 2, p. 25 in [3].)

The above seems to be a very difficult question and we content ourselves with

Received by the editors March 1, 1971.

AMS 1970 subject classifications. Primary 55A35; Secondary 55A10, 57A10.

Key words and phrases. 3-manifold, proper Dehn map, embedding.

Copyright (C) 1972, American Mathematical Society 
the following restricted question: What are sufficient conditions on the $f$ above so that the required $F_{1}$ and $g$ will exist, genus $\left(F_{1}\right)=$ genus $(F)$, and $g$ bd $\left(F_{1}\right)=f$ bd $(F)$ ?

Since $F_{1}$ is to be homeomorphic to $F$, we may drop the subscript 1 .

We shall ask also that $g_{*} \pi_{1}(F) \subset f_{*} \pi_{1}(F)$.

It seems natural to ask that for each component $\mathscr{C}$ of bd $(F), f(\mathscr{C})$ is not nullhomotopic in $M$. We will now obtain sufficient conditions for the existence of the embedding $g$ by

(1) assuming that $g$ exists;

(2) abstracting algebraic and geometric properties of $g$.

We observe that if $g(F)$ (which is necessarily a two-sided surface in $M$ ) is not incompressible in $M$ (that is, $\pi_{1}(g(F)) \rightarrow \pi_{1}(M)$ is not 1-1), it will follow from the loop theorem in [4] that the genus of $g(F)$ could have been reduced. Thus it seems natural to require that $f_{*}: \pi_{1}(F) \rightarrow \pi_{1}(M)$ is $1-1$.

We observe that each component of $f$ bd $(F)$ separates a regular neighborhood of itself in bd $(M)$ since $M$ is orientable.

We consider two possibilities as:

Case 1. $g(F)$ separates $M$;

Case 2. $g(F)$ does not separate $M$.

Case 1. If an embedded incompressible surface $g(F)$ separates $M$ into two 3-submanifolds $M_{1}$ and $M_{2}$, we obtain the group diagram shown in Figure 1 where the $h_{i}$ are homomorphisms induced by inclusion for $i=1, \ldots, 4$.

Since $g_{*}: \pi_{1}(F) \rightarrow \pi_{1}(M)$ is an injection, $h_{1}$ and $h_{2}$ are monomorphisms. If $h_{i}$ was an epimorphism for $i=1$ or 2 , it would follow from 3.1 in [1] that $M_{i}$ was the

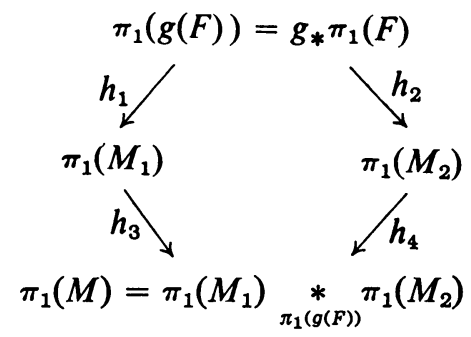

FIGURE 1

product of $F$ with the unit interval since $M$ is irreducible. This case is not so interesting as $g(F)$ could then be deformed to lie in the boundary of $M$. Note that in this case bd $(g(F))$ separates bd $(M)$ into two sets $S_{i}=$ bd $\left(M_{i}\right) \cap$ bd $(M)$ for $i=1,2$. We observe that bd $\left(M_{i}\right)$ is homeomorphic to $S_{i} \cup g(F)$ and that if $S_{i}$ $\cup g(F)$ is connected and we assume a base point on bd $(g(F))$, the image of the map $\pi_{1}\left(S_{i} \cup g(F)\right) \rightarrow \pi_{1}(M)$ is contained in $\pi_{1}\left(M_{i}\right)$ for $i=1,2$.

Let $x$ be a point in bd $(F)$. We shall say that a proper Dehn map $f: F \rightarrow M$ splits $M$ if the following three conditions hold:

(1) $f_{*} \pi_{1}(F, x) \rightarrow \pi_{1}(M, f(x))$ is $1-1$. 
(2) $f$ bd $(F)$ separates bd $(M)$ into two compact, bounded, (not necessarily connected) 2-manifolds $S_{1}$ and $S_{2}$ such that

$$
\begin{aligned}
& S_{1} \cup S_{2}=\text { bd }(M) ; \\
& S_{1} \cap S_{2}=f \text { bd }(F) ; \\
& S_{i} \cup f(F) \text { is connected for } i=1,2 .
\end{aligned}
$$

(3) Let $\bar{S}_{i}$ be the closed surface defined by joining a surface homeomorphic to $S_{i}$ to $F$ along the boundaries of the surfaces so as to make the natural map $\gamma_{i}: \bar{S}_{i} \rightarrow M$ defined by $\gamma_{i} \mid F=f$ and $\gamma_{i} \mid S_{i}=$ the natural inclusion map continuous for $i=1,2$. There exist proper subgroups $A_{1}$ and $A_{2}$ of $\pi_{1}(M, f(x))$ such that the diagram in Figure 2 is commutative.

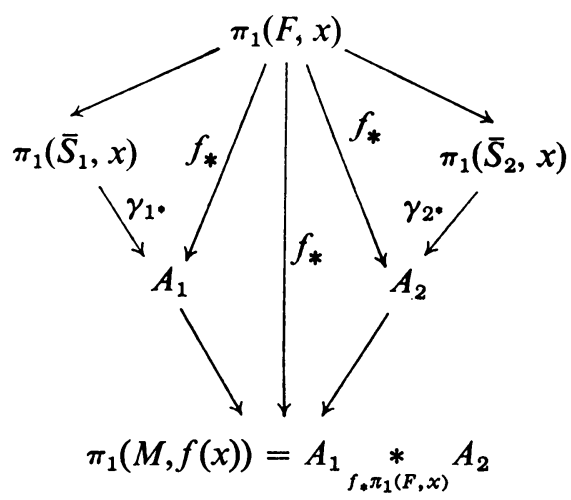

FIGURE 2

We note that the requirement in (2) above, that $S_{i} \cup f(F)$ is connected, is not necessary in our proof. This requirement was introduced to simplify Figure 2.

If $L_{j}$ is a component of $S_{i} \cup f(F)$ which does not meet $f(F)$, one requires that the image of the homomorphism $\pi_{1}\left(L_{\jmath}\right) \rightarrow \pi_{1}(M)$ be conjugate to a subgroup of $A_{i}$ where $i=1$ or 2 .

Case 2. Suppose $g(F)$ does not separate $M$ and $g$ bd $(F)$ does not separate at least one component of bd $(M)$ which it meets. Let $\lambda$ be a simple loop in bd $(M)$ which meets $g$ bd $(F)$ in a single point $x$ and which crosses $g$ bd $(F)$ at that point. We suppose that $g$ bd $(F)$ meets each component of bd $(M)$. Let $A \subset \pi_{1}(M, x)$ be the subgroup of $\pi_{1}(M, x)$ generated by loops based at $x$ and not crossing $g(F)$. It is easily shown that $\pi_{1}(M, x)$ is generated by the elements of $A$ together with $[\lambda]$. Let $S$ be the compact, bounded, 2-manifold obtained by cutting bd $(M)$ along $g$ bd $(F)$. Let $\bar{S}$ be the connected, closed 2-manifold obtained by joining two copies $F^{\prime}, F^{\prime \prime}$ of $F$ to $S$ along their boundaries so that the map $\gamma$ defined by

(1) $\gamma\left|F^{\prime}=g\right| F$;

(2) $\gamma\left|F^{\prime \prime}=g\right| F$;

(3) $\gamma \mid S=$ the natural inclusion map; is continuous.

Now $\gamma_{*} \pi_{1}(\bar{S}, x)$ is a subgroup of $A$. Let $\alpha=\gamma^{-1}(\lambda)$. Then $\alpha$ is a simple arc from 
$F^{\prime}$ to $F^{\prime \prime}$. It is a consequence of Van Kampen's theorem that $\pi_{1}(M, x)$ is the group generated by $A$ and $[\lambda]$ and having all relations of the form

$$
\left[(\gamma \alpha) g(l)(\gamma \alpha)^{-1}\right]=[\lambda][g(l)][\lambda]^{-1}
$$

where $\lambda$ is parameterized appropriately, and $l$ is an arbitrary loop on $F$.

We shall say that a proper Dehn map $f: F \rightarrow M$ cuts $M$ if the following three conditions hold:

(1) $f_{*}: \pi_{1}(F) \rightarrow \pi_{1}(M)$ is $1-1$.

(2) $f$ bd $(F)$ meets each component of bd $(M)$ and fails to separate at least one of them.

(3) Let $\alpha, \lambda, x, \bar{S}$ be as above. Let $A$ be a subgroup of $\pi_{1}(M, x)$. Let $\gamma: \bar{S} \rightarrow M$ be defined by

$$
\gamma\left|F^{\prime}=f ; \quad \gamma\right| F^{\prime \prime}=f ; \quad \gamma \mid S=\text { the natural inclusion map. }
$$

Now we require that $\gamma_{*} \pi_{1}(\bar{S}, x) \subset A$.

We also require that $\pi_{1}(M, x)$ is generated by the elements of $A$ together with $[\lambda]$ and that the relations $\left[(\gamma \alpha) f(l)(\gamma \alpha)^{-1}\right]=[\lambda][f(l)][\lambda]^{-1}$, where $l$ is allowed to be any loop on $F$ based at $x$, completely determine the presentation of $\pi_{1}(M, x)$.

II. Our main theorem. We are now in a position to conveniently state our result.

THEOREM. Let $M$ be a compact, connected, orientable, irreducible 3-manifold. Let $F$ be a compact, connected, orientable 2-manifold with nonvacuous boundary. Let $f: F \rightarrow M$ be a proper Dehn map.

(1) Suppose $f$ splits $M$. Then there is a proper embedding $g: F \rightarrow M$ splitting $M$ such that $g$ bd $(F)=f$ bd $(F)$.

(2) Suppose $f$ cuts $M$. Then there is a proper embedding $g: F \rightarrow M$ cutting $M$ such that $g$ bd $(F)=f$ bd $(F)$.

Furthermore, in both cases $g_{*} \pi_{1}(F)=f_{*} \pi_{1}(F)$.

The idea of the proof is very simple. We construct a complex $X$ having the same fundamental group as $M$. The $X$ we construct will contain an incompressible embedding of $F$ and also an embedding of bd $(M)$. We will then construct a map $G$ from $M$ to $X$ such that $G_{*}: \pi_{1}(M) \rightarrow \pi_{1}(X)$ is an isomorphism and $G \mid \mathrm{bd}(M)$ is a homeomorphism. Using Lemma 1.1 in [6], we will be able to assume $G^{-1}(F)$ is an incompressible surface in $M$. Theorem 1 in [2] will show that $G$ may be taken to be a homeomorphism on this surface. At this point in the proof it will be obvious that $G^{-1}(F)$ is the desired embedding of $F$. We point out that the only places we used the orientability of $M$ were:

(1) to satisfy the conditions of Lemma 1.1 in [6];

(2) to insure that $f$ bd $(F)$ separated a regular neighborhood of itself in bd $(M)$.

We also point out that the assumption that $F$ be orientable is necessary for similar reasons and that if we prove an analog to Lemma 1.1 in [6] for nonorientable 3manifolds and nonorientable 2-manifolds we can easily state a generalization of our theorem covering these cases. 
Proof. We divide the proof into two cases.

Case 1. $f$ separates $M$.

Let $\left(M_{A_{i}}, \rho_{i}\right)$ be the covering space of $M$ associated with the subgroup $A_{i}$ of $\pi_{1}(M, f(x))$ for $i=1,2$. Since $f_{*} \pi_{1}(F, x) \subset A_{i}$ for $i=1,2$, we can find maps $f^{i}, \delta^{i}$ for $i=1,2$ which complete the diagram of maps given in Figure 3 .

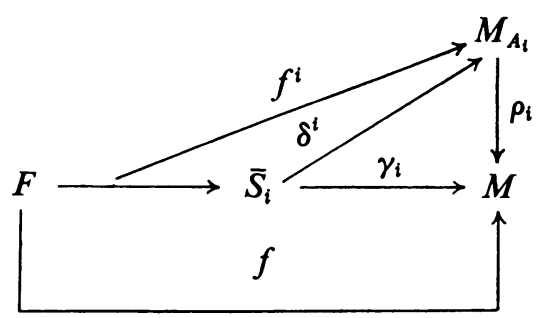

FIGURE 3

Let $X_{1}=M_{A_{1}} \cup F \times\left[0, \frac{1}{2}\right], f^{1}(v)=(v, 0)$ for $v \in F$.

Let $X_{2}=M_{A_{2}} \cup F \times\left[\frac{1}{2}, 1\right], f^{2}(v)=(v, 1)$ for $v \in F$.

Let $X=X_{1} \cup X_{2}$.

Define a map $\rho: X \rightarrow M$ by

(1) $\rho(s)=\rho_{i}(s)$ for $s \in M_{A_{i}}, i=1,2$;

(2) $\rho(v, t)=f(v)$ for $v \in F$ and $t \in[0,1]$.

Then $\rho_{*}$ is easily shown to be an isomorphism. Since $M$ is irreducible, it follows from 1.1.5 in [7] that $\pi_{j}(M)=0$ for $j \geqq 2$.

Now $\pi_{j}\left(M_{A_{i}}\right)=\pi_{j}\left(X_{i}\right)=0$ for $j \geqq 2, i=1,2$, and by Theorem 5, p. 199 in [5], $\pi_{j}(X)=0$ for $j \geqq 2$. Let

$$
S^{*}=\left(\delta^{1}\left(\bar{S}_{1}\right)-f^{1}(\operatorname{int}(F))\right) \cup\left(\delta^{2}\left(\bar{S}_{2}\right)-f^{2}(\operatorname{int}(F))\right) \cup(\text { bd }(F) \times[0,1]) .
$$

Now $S^{*}$ is homeomorphic to bd $(M)$ and $\rho S^{*}=$ bd $(M)$. In fact,

$$
\rho \mid\left(S^{*}-(\operatorname{bd}(F) \times[0,1])\right)
$$

is a homeomorphism. Also,

$$
\rho_{*} \pi_{1}\left(S^{*}, f^{1}(x)\right)=\pi_{1}(\text { bd }(M), f(x)) \subset \pi_{1}(M, f(x)) .
$$

We now define a map $G:(\operatorname{bd}(M), f(x)) \rightarrow\left(S^{*}, f^{1}(x)\right)$ so that $\rho_{*} G_{*}$ : $\pi_{1}(\mathrm{bd}(M), f(x)) \rightarrow \pi_{1}(\mathrm{bd}(M), f(x))$ is the identity. Since $\pi_{j}(X)=0$ for $j \geqq 2$, it is a simple matter to extend $G$ to $M$ so that $G_{*}=\rho_{*}^{-1}: \pi_{1}(M) \rightarrow \pi_{1}(X)$ and $G^{-1}\left(S^{*}\right)$ $=$ bd $(M)$. This is done by first extending $G$ to the 1 -skeleton of $M$, then to the 2-skeleton, and finally to all 3-simplexes.

It is a consequence of Lemma 1.1 in [6] that $G^{-1}\left(F \times\left\{\frac{1}{2}\right\}\right)$ can be taken to be an incompressible surface in $M$. Let $F_{1}$ be a component of this surface. Since $G_{*}$ is an isomorphism, $\left(G \mid F_{1}\right)_{*}: \pi_{1}\left(F_{1}\right) \rightarrow \pi_{1}\left(F \times\left\{\frac{1}{2}\right\}\right)$ is a monomorphism. Observe that $F_{1}$ is not closed since $\pi_{1}(F)$ is free. It follows from Theorem 1 in [2] that either

(a) $G \mid F_{1}$ is homotopic, leaving bd $\left(F_{1}\right)$ fixed, to a covering map, or 
(b) $F_{1}$ is an annulus or möbius band and $G \mid F_{1}$ is homotopic to a map carrying $F_{1}$ to bd $\left(F \times\left\{\frac{1}{2}\right\}\right)$.

We observe that $G \mid$ bd $\left(F_{1}\right)$ is a homeomorphism by construction. Thus if $F_{1}$ is an annulus so is $F \times\left\{\frac{1}{2}\right\}$ and $G \mid F_{1}$ is homotopic to a homeomorphism leaving bd $\left(F_{1}\right)$ fixed. Since $F_{1}$ is assumed to separate a regular neighborhood of itself and $M$ is orientable, $F_{1}$ is not a möbius band. It follows that $G \mid F_{1}$ is homotopic to a covering map leaving bd $\left(F_{1}\right)$ fixed. Since $G \mid$ bd $\left(F_{1}\right)$ is a homeomorphism, $G \mid F_{1}$ is homotopic to a homeomorphism leaving bd $\left(F_{1}\right)$ fixed.

It follows that we may assume that $G \mid F_{1}$ is a homeomorphism. Since $F$ is connected, $G^{-1}\left(F \times\left\{\frac{1}{2}\right\}\right)=F_{1}$. Thus we can find an embedding $g: F \rightarrow F_{1}$. Let $M_{i}=G^{-1}\left(X_{i}\right)$ for $i=1,2$.

Now, by construction, $G_{*} \pi_{1}\left(M_{i}, f(x)\right) \subseteq \pi_{1}\left(X_{i}, G f(x)\right)=A_{i}$ for $i=1,2$; and since $G \mid g(F)$ is a homeomorphism, $G_{*} \pi_{1}(g(F), f(x))=\pi_{1}\left(F \times\left\{\frac{1}{2}\right\}, G f(x)\right) \subset \pi_{1}(X, G f(x))$.

It follows from Proposition 2 in [1] that $G_{*} \pi_{1}\left(M_{i}, f(x)\right)=\pi_{1}\left(X_{i}, G f(x)\right)$ for $i=1,2$ and that the splitting induced by the embedding $g$ is the same as that induced by our original map $f$.

Case 2. $f(F)$ cuts $M$.

Let $\left(M_{A}, \rho_{1}\right)$ be the covering space of $M$ associated with $A \subset \pi_{1}(M, x)$.

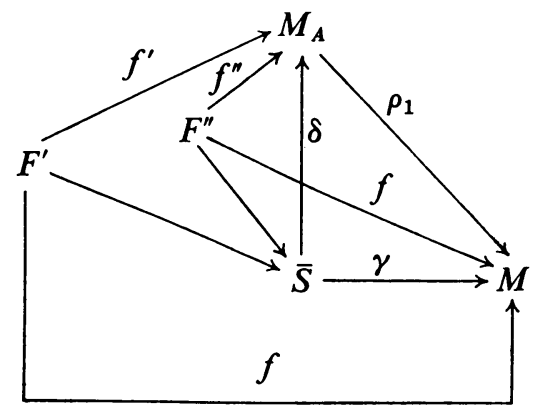

Figure 4

Since $\gamma_{*} \pi_{1}(\bar{S}, x) \subset A$, one can find the maps $f^{\prime}, f^{\prime \prime}$ and $\delta$ necessary to complete the diagram of maps in Figure 4. Let $X$ be the complex defined by $X=M_{A} \cup F$ $\times[0,1]$ where we make the identifications $(v, 0)=f^{\prime}(v)$ for $v$ in $F$ and $(v, 1)=f^{\prime \prime}(v)$ for $v$ in $F$.

Define a map $\bar{\rho}: X \rightarrow M$ by

(1) $\bar{\rho} \mid M_{A}=\rho_{1}$;

(2) $\bar{\rho}(s, t)=f(s)$ for $s$ in $F$ and $t$ in $[0,1]$.

Now $\bar{\rho}_{*}$ has been constructed to be an isomorphism. One sees this by picking the elements of $\pi_{1}\left(M_{A}\right) \subset \pi_{1}(X)$ and an element representing the loop $\bar{\rho}^{-1}(\lambda)$ as generators for $\pi_{1}(X)$. The relations are the natural ones among the elements of $\pi_{1}\left(M_{A}\right)$ together with a collection of relations which are carried by $\bar{\rho}$ to the relations mentioned in $(* *)$. 
Now $\pi_{j}\left(M_{A}\right)=0$ for $j \geqq 2$ since $\pi_{j}(M)=0$ by 1.1.5 in [7]. We wish to show that $\pi_{j}(X)=0$ for $j \geqq 2$. Let $X /$ be the space obtained by cutting $X$ along $F \times\left\{\frac{1}{2}\right\}$. Let $F_{1}$ and $F_{2}$ be disjoint surfaces in $X /$ coming from $F \times\left\{\frac{1}{2}\right\}$. We can form an infinite cyclic covering $\left(X^{*}, \rho\right)$ of $X$ by taking infinitely many copies $X_{l}^{k}$ of $X_{l}$ and pasting $F_{1}^{k}$ to $F_{2}^{k+1}$ for $k$ an integer and $F_{i}^{k}$ the copy of $F_{i}$ contained in $X_{1}^{k}$ for $i=1,2$. We claim that $\pi_{j}\left(X^{*}\right)=0$ for $j \geqq 2$ and thus $\pi_{j}(X)=0$ for $j \geqq 2$. We observe that it follows from Theorem 5, p. 199 in [5] that $\pi_{j}\left(\bigcup_{k=l}^{l+N} X_{l}^{k}\right)=0$ where $l$ is an integer and $N$ is a positive integer for $j \geqq 2$ since $M_{A}$ is a deformation retract of $X_{l}$. However, since a sphere is compact, the image of a sphere in $X^{*}$ meets only finitely many of the $F_{i}^{k}$ and thus lies in a set of the form $\bigcup_{k=l}^{l+N} X_{l}^{k}$ where $l$ is an integer and $N$ is a positive integer. It follows that $\pi_{j}\left(X^{*}\right)=0$ for $j \geqq 2$ and thus that $\pi_{j}(X)=0$ for $j \geqq 2$.

Let $\bar{S}^{\prime}$ be the closure of $\bar{S}-\left(F^{\prime} \cup F^{\prime \prime}\right)$. Then the surface $\delta\left(\bar{S}^{\prime}\right) \cup$ bd $(F) \times[0,1]$ is homeomorphic to bd $(M)$.

As was done in Case 1 we can find a map $G: M \rightarrow X$ such that

(1) $G$ bd $(M)=\delta\left(\bar{S}^{\prime}\right) \cup$ bd $(F) \times[0,1]$.

(2) $G \mid \mathrm{bd}(M)$ is a homeomorphism.

(3) $G^{-1} G$ bd $(M)=$ bd $(M)$.

(4) $\tilde{\rho}_{*} G_{*}: \pi_{1}(M) \rightarrow \pi_{1}(M)$ is the identity.

It now follows from Lemma 1.1 in [6] that there is a map $H$ homotopic to $G$ such that

(a) $H \mid$ bd $(M)=G \mid$ bd $(M)$.

(b) $H$ satisfies the three conditions on $G$ given above.

(c) $H^{-1}\left(F \times\left\{\frac{1}{2}\right\}\right)$ is an incompressible surface $\mathscr{F}$ in $M$.

It follows from Theorem 1 in [2] that $H \mid \mathscr{F}$ may be taken to be a homeomorphism. Thus $\mathscr{F}=F$.

Note $\pi_{1}(M) \supset \pi_{1}(\mathscr{F})=f_{*} \pi_{1}(F)$.

This completes the proof of our theorem.

\section{BiBLIOGRAPHY}

1. E. M. Brown, Unknotting in $M^{2} \times I$, Trans. Amer. Math. Soc. 123 (1966), 480-505. MR 33 \#6640.

2. W. Heil, On $P^{2}$-irreducible 3-manifolds, Bull. Amer. Math. Soc. 75 (1969), 772-775. MR 40 \#4958.

3. C. D. Papakyriakopoulos, On Dehn's lemma and the asphericity of knots, Ann. of Math. (2) 66 (1957), 1-26. MR 19, 761.

4. J. Stallings, On the loop theorem, Ann. of Math. (2) 72 (1960), 12-19. MR 22 \#12526.

5. The mathematical works of J. H. C. Whitehead, Vol. 2, Macmillan, New York, 1963.

6. F. Waldhausen, Gruppen mit Zentrum und 3-dimensionale Mannigfaltigkeiten, Topology 6 (1967), 505-517. MR 38 \#5223.

7. - On irreducible 3-manifolds which are sufficiently large, Ann. of Math. (2) 87 (1968), 56-88. MR 36 \#7146.

Institute for Defense Analyses, Princeton, New Jersey 08540

Current address: Virginia Polytechnic Institute and State University, Blacksburg, Virginia 24601 